\title{
Local Proof of Algebraic Characterization of Free Actions ${ }^{\star}$
}

Paul F. BAUM ${ }^{\dagger \ddagger}$ and Piotr M. HAJAC $\ddagger \S$

${ }^{\dagger}$ Mathematics Department, McAllister Building, The Pennsylvania State University, University Park, PA 16802, USA

E-mail: baum@math.psu.edu

‡ Instytut Matematyczny, Polska Akademia Nauk, ul. Śniadeckich 8, Warszawa, 00-656 Poland E-mail: pmh@impan.pl

URL: http://www.impan.pl/ pmh

$\S$ Katedra Metod Matematycznych Fizyki, Uniwersytet Warszawski, ul. Hoża 74, 00-682 Warszawa, Poland

Received February 13, 2014, in final form May 21, 2014; Published online June 06, 2014 http://dx.doi.org/10.3842/SIGMA.2014.060

\begin{abstract}
Let $G$ be a compact Hausdorff topological group acting on a compact Hausdorff topological space $X$. Within the $C^{*}$-algebra $C(X)$ of all continuous complex-valued functions on $X$, there is the Peter-Weyl algebra $\mathcal{P}_{G}(X)$ which is the (purely algebraic) direct sum of the isotypical components for the action of $G$ on $C(X)$. We prove that the action of $G$ on $X$ is free if and only if the canonical map $\mathcal{P}_{G}(X) \otimes_{C(X / G)} \mathcal{P}_{G}(X) \rightarrow \mathcal{P}_{G}(X) \otimes \mathcal{O}(G)$ is bijective. Here both tensor products are purely algebraic, and $\mathcal{O}(G)$ denotes the Hopf algebra of "polynomial" functions on $G$.
\end{abstract}

Key words: compact group; free action; Peter-Weyl-Galois condition

2010 Mathematics Subject Classification: 22C05; 55R10; 57S05; 57S10

With admiration and affection, to Marc A. Rieffel on the occasion of his 75th birthday

\section{Theorem}

Given a compact Hausdorff topological group $G$, we denote by $\mathcal{O}(G)$ the dense Hopf $*$-subalgebra of the commutative $C^{*}$-algebra $C(G)$ spanned by the matrix coefficients of irreducible representations of $G$. Let $X$ be a compact Hausdorff topological space with a given continuous (right) action of $G$. The action map

$$
X \times G \ni(x, g) \longmapsto x g \in X
$$

determines a map of $C^{*}$-algebras

$$
\delta: C(X) \longrightarrow C(X \times G) .
$$

Moreover, denoting by $\otimes$ the purely algebraic tensor product over the field $\mathbb{C}$ of complex numbers, we define the Peter-Weyl subalgebra [2, (3.1.4)] of $C(X)$ as

$$
\mathcal{P}_{G}(X):=\{a \in C(X) \mid \delta(a) \in C(X) \otimes \mathcal{O}(G)\} .
$$

Using the coassociativity of $\delta$, one can check that $\mathcal{P}_{G}(X)$ is a right $\mathcal{O}(G)$-comodule algebra. In particular, $\mathcal{P}_{G}(G)=\mathcal{O}(G)$. The assignment $X \mapsto \mathcal{P}_{G}(X)$ is functorial with respect to continuous

\footnotetext{
${ }^{\star}$ This paper is a contribution to the Special Issue on Noncommutative Geometry and Quantum Groups in honor of Marc A. Rieffel. The full collection is available at http://www.emis.de/journals/SIGMA/Rieffel.html
} 
$G$-equivariant maps and comodule algebra homomorphisms. We call it the Peter-Weyl functor. Equivalently [10, Proposition 2.2], $\mathcal{P}_{G}(X)$ is the (purely algebraic) direct sum of the isotypical components for the action of $G$ on $C(X)$ (see [7, p. 31] and [6], cf. [8, Theorem 1.5.1]). In the special case that the action of $G$ on $X$ is free, $\mathcal{P}_{G}(X)$ is the algebra of all continuous sections of the vector bundle $E$ on $X / G$, where

$$
E:=X \underset{G}{\times} \mathcal{O}(G)
$$

Note that in forming this vector bundle, $\mathcal{O}(G)$ is topologized as the direct limit of its finitedimensional vector subspaces, not by the norm topology.

The theorem of this paper is:

Theorem 1. Let $X$ be a compact Hausdorff space equipped with a continuous (right) action of a compact Hausdorff group $G$. Then the action is free if and only if the canonical map

$$
\begin{aligned}
& \text { can }: \mathcal{P}_{G}(X) \underset{C(X / G)}{\otimes} \mathcal{P}_{G}(X) \longrightarrow \mathcal{P}_{G}(X) \otimes \mathcal{O}(G), \\
& \text { can }: x \otimes y \longmapsto(x \otimes 1) \delta(y),
\end{aligned}
$$

is bijective. (Here both tensor products are purely algebraic.)

Definition 1. The action of a compact Hausdorff group $G$ on a compact Hausdorff space $X$ satisfies the Peter-Weyl-Galois $(P W G)$ condition iff the canonical map (2) is bijective.

Our result states that the usual formulation of free action is equivalent to the algebraic PWGcondition. In particular, our result provides a framework for extending Chern-Weil theory beyond the setting of differentiable manifolds and into the context of cyclic homology - noncommutative geometry [3].

\section{Proof}

The proof of the equivalence of freeness and the PWG-condition consists of six steps. The first step takes care of the easy implication of the equivalence, and the remaining five steps prove the difficult implication of the equivalence.

\section{1 $\quad$ PWG-condition $\Rightarrow$ freeness}

It is immediate that the action is free, i.e. $x g=x \Longrightarrow g=e$ (where $e$ is the identity element of $G$ ), if and only if

$$
\begin{aligned}
& F: X \times G \longrightarrow X \underset{X / G}{\times} X, \\
& F:(x, g) \longmapsto(x, x g),
\end{aligned}
$$

is a homeomorphism. Here $X \times_{X / G} X$ is the subset of $X \times X$ consisting of pairs $\left(x_{1}, x_{2}\right)$ such that $x_{1}$ and $x_{2}$ are in the same $G$-orbit.

This is equivalent to the assertion that the $*$-homomorphism

$$
F^{*}: C(X \underset{X / G}{\times} X) \longrightarrow C(X \times G)
$$


obtained from the above map $F$ is an isomorphism. Note that $F$ is always surjective, so that the $*$-homomorphism $F^{*}$ is always injective. Furthermore, there is the following commutative diagram in which the vertical arrows are the evident maps:

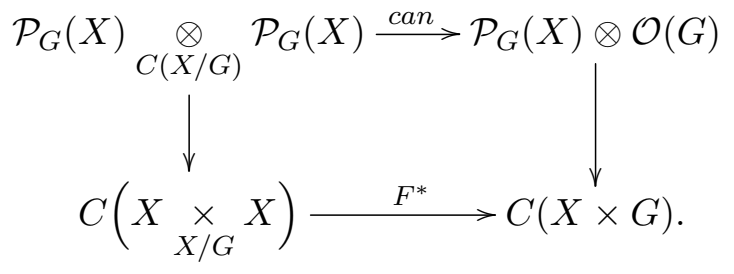

Since the right-hand side of the canonical map (2), i.e. $\mathcal{P}_{G}(X) \otimes \mathcal{O}(G)$, is dense in the $C^{*}$-algebra $C(X \times G)$, validity of the PWG-condition combined with the commutativity of the diagram (3) implies that the image of the $*$-homomorphism $F^{*}$ is dense in $C(X \times G)$. Therefore, as the image of a $*$-homomorphism of $C^{*}$-algebras is always closed, PWG implies surjectivity of $F^{*}$, which in turn implies that the action of $G$ on $X$ is free.

\subsection{Reduction to surjectivity and matrix coefficients}

Lemma 1. Let $X$ be a compact Hausdorff space equipped with a continuous (right) action of a compact Hausdorff group $G$. Then the canonical map is surjective if and only if for any matrix coefficient $h$ of an irreducible representation of $G$, the element $1 \otimes h$ is in the image of the canonical map. Moreover, if the canonical map is surjective, then it is bijective.

Proof. First observe that the canonical map is a homomorphism of left $\mathcal{P}_{G}(X)$-modules. The first assertion of the lemma follows by combining this observation with the fact that matrix coefficients of irreducible representations span $\mathcal{O}(G)$ as a vector space.

The Hopf algebra $\mathcal{O}(G)$ is cosemisimple. Hence, by the result of H.-J. Schneider [9, Theorem I], if the canonical map is surjective, then it is bijective.

Alternately, assuming that the action of $G$ on $X$ is free, injectivity can be directly proved by using the vector-bundle point of view indicated in (1) (see [1]).

\subsection{Reduction to free actions of compact Lie-groups}

Assume that Theorem 1 holds for compact Lie groups. In this section, we prove that this special case implies Theorem 1 in general.

Let $\varphi: G \rightarrow U(n)$ be any finite-dimensional representation of $G$. Set

$$
X_{\varphi}:=X \underset{G}{\times} U(n) .
$$

Thus $X_{\varphi}=(X \times U(n)) / G$, where $G$ acts on $X \times U(n)$ by $(x, u) g:=\left(x g, \varphi\left(g^{-1}\right) u\right)$. The group $U(n)$ acts on $X_{\varphi}$ by $[(x, u)] v:=[(x, u v)]$, and this action is free. The map

$$
\Phi: X \longrightarrow X_{\varphi}, \quad x \longmapsto\left[\left(x, I_{n}\right)\right]
$$

where $I_{n} \in U(n)$ is the identity matrix, has the equivariance property

$$
\Phi(x g)=\Phi(x) \varphi(g)
$$


Hence $\Phi$ and $\varphi$ induce maps $\Phi^{*}: \mathcal{P}_{U(n)}\left(X_{\varphi}\right) \rightarrow \mathcal{P}_{G}(X)$ and $\varphi^{*}: \mathcal{O}(U(n)) \rightarrow \mathcal{O}(G)$. The equivariance property (4) implies commutativity of the diagram

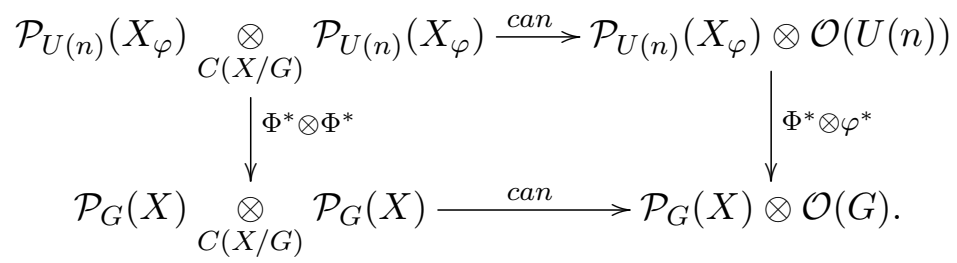

Therefore surjectivity of the upper canonical map implies that $1 \otimes h$ is in the image of the lower canonical map, where $h$ is any matrix coefficient of $\varphi$. By Lemma 1, this implies the PWG-condition.

\subsection{Local triviality for free actions of compact Lie groups}

We recall the theorem of A.M. Gleason:

Theorem 2 ([4]). Let $G$ be a compact Lie group acting freely and continuously on a completely regular space $X$. Then $X$ is a locally trivial $G$-bundle over $X / G$.

Combining the Gleason theorem with Section 2.3, we infer that the PWG-condition is valid for free actions if it is valid for locally trivial free actions.

\subsection{Reduction to the trivial-bundle case}

Assume that the action of $G$ on $X$ is free and locally trivial. Since the quotient space $X / G$ is compact Hausdorff, we can choose a finite open cover $U_{1}, \ldots, U_{r}$ of $X / G$ such that each $\pi^{-1}\left(U_{j}\right)$ is a trivializable principal $G$-bundle over $U_{j}$. Here $\pi: X \rightarrow X / G$ is the quotient map. On $X / G$, let $\psi_{1}, \ldots, \psi_{r}$ be a partition of unity subordinate to the cover $U_{1}, \ldots, U_{r}$. Then, for each $j$ there is the canonical map

$$
\operatorname{can}_{j}: \mathcal{P}_{G}\left(\pi ^ { - 1 } ( \operatorname { s u p p } ( \psi _ { j } ) ) \underset { C ( \operatorname { s u p p } ( \psi _ { j } ) ) } { \otimes } \mathcal { P } _ { G } \left(\pi ^ { - 1 } ( \operatorname { s u p p } ( \psi _ { j } ) ) \longrightarrow \mathcal { P } _ { G } \left(\pi^{-1}\left(\operatorname{supp}\left(\psi_{j}\right)\right) \otimes \mathcal{O}(G)\right.\right.\right.
$$

Assume that for each $j \in\{1, \ldots, r\}$ there exist elements

$$
p_{j 1}, q_{j 1}, \ldots, p_{j n}, q_{j n} \in \mathcal{P}_{G}\left(\pi^{-1}\left(\operatorname{supp}\left(\psi_{j}\right)\right) \quad \text { such that } \quad \operatorname{can}_{j}\left(\sum_{i=1}^{n} p_{j i} \otimes q_{j i}\right)=1 \otimes h\right.
$$

Let $\widetilde{p_{j i}}$ 's and $\widetilde{q_{j i}}$ 's be functions on $X$ obtained respectively from functions $p_{j i}$ 's and $q_{j i}$ 's by zero-value extension. Then for each $i$ and $j$ we take

$$
\widetilde{p_{j i}} \sqrt{\psi_{j} \circ \pi}, \widetilde{q_{j i}} \sqrt{\psi_{j} \circ \pi} \in \mathcal{P}_{G}(X),
$$


and for any $x \in X$ and $g \in G$, using the commutativity of the diagram (3), we obtain

$$
\begin{aligned}
\operatorname{can}( & \left.\sum_{j=1}^{r} \sum_{i=1}^{n} \widetilde{p_{j i}} \sqrt{\psi_{j} \circ \pi} \otimes \widetilde{q_{j i}} \sqrt{\psi_{j} \circ \pi}\right)(x, g) \\
= & \left(\sum_{j=1}^{r} \sum_{i=1}^{n} \widetilde{p_{j i}} \sqrt{\psi_{j} \circ \pi} \otimes \widetilde{q_{j i}} \sqrt{\psi_{j} \circ \pi}\right)(x, x g) \\
= & \sum_{\text {all } j \text { s.t. } \pi(x) \in U_{j}}\left(\psi_{j} \circ \pi\right)(x) \sum_{i=1}^{n} p_{j i}(x) q_{j i}(x g) \\
= & \sum_{\text {all } j \text { s.t. } \pi(x) \in U_{j}}\left(\psi_{j} \circ \pi\right)(x) c a n_{j}\left(\sum_{i=1}^{n} p_{j i} \otimes q_{j i}\right)(x, g) \\
= & \sum_{j=1}^{r}\left(\psi_{j} \circ \pi\right)(x) h(g) \\
= & (1 \otimes h)(x, g) .
\end{aligned}
$$

Hence validity of the PWG-condition in the trivial-bundle case implies that the PWG-condition holds for actions that are free and locally trivial.

\subsection{The trivial-bundle case}

First note that

$$
\mathcal{P}_{G}(Y \times G)=C(Y) \otimes \mathcal{O}(G) .
$$

This is implied by two facts: (1) quite generally $\mathcal{P}_{G}(X) \subseteq C(X)$ is the purely algebraic direct sum of the isotypical components for the action of $\mathrm{G}$ on $C(X)$ [10, Proposition 2.2]; and (2) each isotypical component for the action of $\mathrm{G}$ on $C(Y \times G)$ is of the form $C(Y) \otimes V$, where $V$ is an isotypical component for the action of $G$ on $C(G)$.

As $\mathcal{O}(G)$ is a Hopf algebra, the dual of the homeomorphism

$$
F_{G}: G \times G \ni\left(g_{1}, g_{2}\right) \longmapsto\left(g_{1}, g_{1} g_{2}\right) \in G \times G
$$

and the dual of its inverse $F_{G}^{-1}$ restrict and corestrict respectively to

$$
\begin{aligned}
& \operatorname{can}_{G}: \mathcal{O}(G) \otimes \mathcal{O}(G) \ni T \longmapsto T \circ F_{G} \in \mathcal{O}(G) \otimes \mathcal{O}(G), \\
& \operatorname{can}_{G}^{-1}: \mathcal{O}(G) \otimes \mathcal{O}(G) \ni T \longmapsto T \circ F_{G}^{-1} \in \mathcal{O}(G) \otimes \mathcal{O}(G) .
\end{aligned}
$$

Granted the identification (5), we now obtain the following commutative diagram:

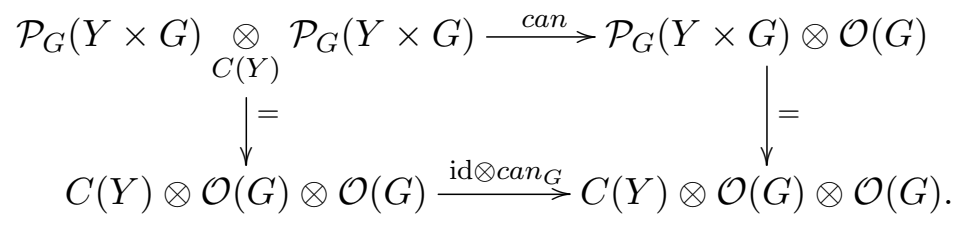

Hence the bijectivity of can $_{G}$ implies the bijectivity of $c a n$. 


\section{Appendix}

In this appendix, we observe that Step 2.5, i.e. reduction to the trivial-bundle case, is implied by the following general results:

Lemma 2. Let $(H, \Delta)$ be a compact quantum group acting on a unital $C^{*}$-algebra $A$. The assignment $A \mapsto \mathcal{P}_{H}(A)$ of the Peter-Weyl algebra to a $C^{*}$-algebra yields a functor from the category with objects being unital $C^{*}$-algebras with an $H$-coaction (and morphisms being equivariant unital ${ }^{*}$-homomorphisms) to the category whose objects are $\mathcal{O}(H)$-comodule algebras (and whose morphisms are colinear algebra homomorphisms). Furthermore, this functor commutes with all (equivariant) pullbacks, that is,

$$
\mathcal{P}_{H}(A \times C)=\mathcal{P}_{H}(A) \underset{\mathcal{P}_{H}(B)}{\times} \mathcal{P}_{H}(C) .
$$

Lemma 3 (Lemma 3.2 in [5]). Let $\mathcal{H}$ be a Hopf algebra with bijective antipode, and let

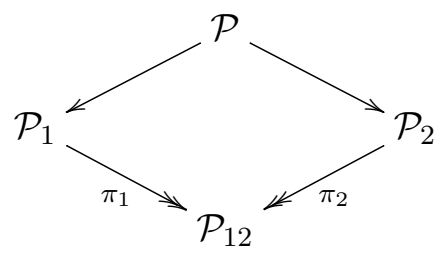

be the pullback diagram of surjective right $\mathcal{H}$-comodule algebra homomorphisms. Then $\mathcal{P}$ is principal if and only if $\mathcal{P}_{1}$ and $\mathcal{P}_{2}$ are principal ${ }^{1}$.

Proving the first lemma is straightforward, and the second lemma is the highlight of [5]. For the case considered in this paper (a compact Hausdorff group $G$ acting continuously on a compact Hausdorff space $X$ ), we have $A=C(X), H=C(G), \mathcal{O}(H)=\mathcal{O}(G), \mathcal{P}_{H}(A)=\mathcal{P}_{G}(X)$, and the condition of being "principal" is equivalent to the PWG-condition. Thus these two lemmas combined with standard induction yield an alternative proof of Step 2.5.

Moreover, the theorem of this paper is a special case of a much more general theorem about compact quantum groups acting on unital $C^{*}$-algebras [1]. However, the proof of the general theorem is nonlocal.

\section{Acknowledgments}

We thank the referees for the careful attention they have given to this paper. This work was partially supported by NCN grant 2011/01/B/ST1/06474. P.F. Baum was partially supported by NSF grant DMS 0701184.

\section{References}

[1] Baum P.F., De Commer K., Hajac P.M., Free actions of compact quantum groups on unital $C^{*}$-algebras, arXiv:1304.2812.

[2] Baum P.F., Hajac P.M., Matthes R., Szymański W., Noncommutative geometry approach to principal and associated bundles, in Quantum Symmetry in Noncommutative Geometry, to appear, math.DG/0701033.

[3] Brzeziński T., Hajac P.M., The Chern-Galois character, C. R. Math. Acad. Sci. Paris 338 (2004), 113-116, math.KT/0306436.

[4] Gleason A.M., Spaces with a compact Lie group of transformations, Proc. Amer. Math. Soc. 1 (1950), 35-43.

\footnotetext{
${ }^{1}$ For the definition of "principal" see [3] and [5, Definition 2.3].
} 
[5] Hajac P.M., Krähmer U., Matthes R., Zieliński B., Piecewise principal comodule algebras, J. Noncommut. Geom. 5 (2011), 591-614, arXiv:0707.1344.

[6] Loomis L.H., An introduction to abstract harmonic analysis, D. Van Nostrand Company, Inc., Toronto New York - London, 1953.

[7] Mostow G.D., Cohomology of topological groups and solvmanifolds, Ann. of Math. 73 (1961), 20-48.

[8] Podleś P., Symmetries of quantum spaces. Subgroups and quotient spaces of quantum SU(2) and $\mathrm{SO}(3)$ groups, Comm. Math. Phys. 170 (1995), 1-20, hep-th/9402069.

[9] Schneider H.-J., Principal homogeneous spaces for arbitrary Hopf algebras, Israel J. Math. 72 (1990), 167195.

[10] Soltan P.M., On actions of compact quantum groups, Illinois J. Math. 55 (2011), 953-962, arXiv:1003.5526. 